Francisco Javier García Díaz ${ }^{1} *$

Mercedes Muñoz Conde 2

Rafael Cabello Jaime ${ }^{3}$

1. Enfermero de Práctica Avanzada en Heridas Crónicas Complejas. Unidad de Gestión Clínica de Algarrobo. Área de Gestión Sanitaria de Algarrobo. Este de Málaga-Axarquía. Málaga. España.

2. Enfermera de Práctica Avanzada en Heridas Crónicas Complejas. Área de Gestión Sanitaria Este de Málaga-Axarquía. Málaga. España.

3. Enfermero en Atención Primaria Experto en heridas. Unidad de Gestión Clínica Vélez-Norte. Área de Gestión Sanitaria. Este de Málaga-Axarquía. Málaga. España.

*Autor para correspondencia.

Correo electrónico: javierirma@hotmail.com (Francisco Javier García Díaz).

Recibido el 18 de noviembre de 2020; aceptado el 21 de diciembre de 2020

\section{Comparación entre el coste y el cierre de heridas en una unidad de gestión clínica que incluye una enfermera de práctica avanzada en heridas crónicas complejas}

\section{Comparison between the cost and the wound closure in a Clinical Management Unit that includes an Advanced Practice Nurse on Complex Chronic Wounds}

\section{RESUMEN}

Introducción: Las heridas de difícil cicatrización o complejas que aquejan a un alto porcentaje de la población, además de afectar a la calidad de vida de quien las padece, repercute en un alto coste económico, en tiempo de enfermería y en estancias hospitalarias.

Objetivo: Conocer el coste económico, las heridas complicadas, el coste medio para el cierre y la media de días para el mismo que ha tenido cada profesional de enfermería comparado con la enfermera de práctica avanzada (EPA) en heridas crónicas complejas (HCC) de la Unidad.

Metodología: Estudio descriptivo longitudinal del total de los informes de valoración de heridas generados desde la aplicación de heridas en DIRAYA (sistema de información asistencial corporativo para todo el Servicio Andaluz de Salud, historia de salud única de todos los episodios asistenciales del ciudadano) y que necesitaron materiales de cura en ambiente húmedo suministrados por la Unidad de Gestión

Clínica (UGC). El estudio se desarrolló en la UGC de Algarrobo, perteneciente al Área de Gestión Sanitaria Este de Málaga-Axarquía, desde junio 2019 hasta junio 2020. Se trata de una muestra conceptual, ya que se ha incluido a toda la población de enfermeros y enfermeras de la UGC y las correspondientes hojas emitidas por ellos. Resultados:

Durante el periodo de estudio se registraron un total de 416 heridas en la Unidad, de las cuales se cerraron 338, el 81,2\%. La EPA trató el $22,6 \%$ del total de las heridas (94 heridas) y resolvió el 90,4\% de ellas, siendo el coste medio para el cierre de las heridas de $21,3 €$, en un plazo medio de 30,7 días, mientras que el resto de profesionales tuvo que tratar de media 32,2 heridas y se resolvieron de media el $76,7 \%$, siendo el coste medio para el cierre de una herida de $36,2 €$ en un plazo medio de 172,1 días. Conclusiones: La introducción de la EPA en HCC en la UGC ha llevado a la Unidad a un mejor resultado en cuanto a gasto en materiales y al acortamiento de los días para la resolución de las heridas, lo que influye en una mejor calidad de vida para aquellos que las padecen. Además, la introducción de la EPA ha servido de revulsivo

\section{ABSTRACT}

Introduction: Hard-to-heal or complex wounds that afflict a high percentage of the population, besides affecting the quality of life of the ones who suffer from them, impacts on a high economic cost, on nursing time and on hospital stays. Aims: Knowing the economic cost, complex wounds, average cost for closing and the average number of days for it that every nurse had compared to the Advanced Practice Nurse (APN) on complex chronic wounds from the unit. Methodology: Longitudinal descriptive study of the total injury assessment reports generated from the wounds application on DIRAYA (corporate healthcare information system for the entire Andalusian health service, unique Health Story of all citizen welfare episodes) and that needed healing material in a humid environment supplied by CMU. The study was developed in the Clinical Management Unit (CMU) of Algarrobo, belonging to the East Health Management Area of Málaga - Axarquía, since June 2019 until June 2020. This is a conceptual sample as the entire CMU nurse population and the corresponding pages issued by them have been included. Results: During the study period, a total of 416 wounds were registered in the Unit, of which 338 were closed, $81.2 \%$. The APN treated $22.6 \%$ of the total wounds (94 wounds) and resolved the $90.4 \%$ of them, being the average cost for the wounds closure of $21.3 €$, within an average of 30.7 days. While the rest of the professionals had to treat an average of 32.2 wounds and the average resolved was $76.7 \%$, being the average cost for the wound closure of $36.2 €$ within an average of 172,1 days. Conclusions: The introduction of the APN on complex chronic wounds in the CMU had led the unit to a better result in terms of spending on materials and shortening the days for the wounds resolution, which influences a better quality of life for those who suffer from them. In addition, the introduction of the APN has 
para los profesionales y les ha llevado a un mejor hacer en su trabajo con las heridas, lo que influye tanto en su autoestima profesional como en los pacientes a los que tratan.

PALABRAS CLAVE: Cicatrización de lesiones, heridas complejas, enfermera de práctica avanzada, costes. served as a stimulus for professionals, leading them to do a better job with the wound, which influences both their professional self-esteem and as well as the patients they treat.

KEYWORDS: Wound healing, complicated wounds, advanced practice nursing, cost.

\section{У INTRODUCCIÓN}

Diferentes estudios han puesto de manifiesto el impacto económico que supone para el sistema sanitario el proceso de curación de las distintas heridas. Nadie puede negar hoy en día que el gasto en materiales de cura en ambiente húmedo y de apósitos en heridas de difícil cicatrización, lesiones relacionadas con la dependencia y úlceras por presión (UPP), sin olvidarnos las de origen circulatorio o derivadas de enfermedades metabólicas como la diabetes, supone un desgaste económico en materiales y tiempo de profesionales, sin contar los costes indirectos como la pérdida de productividad o la disminución de la calidad de vida.

Hoy en día se tiende a denominar las heridas crónicas o de difícil cicatrización como heridas complejas, ya que el término crónico hace referencia a procesos que no se solucionan y acompañan a la persona a lo largo de su vida ${ }^{1}$.

Según distintos estudios, el coste de la atención a heridas complejas puede representar un 22\% de gasto en apósitos, un $41 \%$ en tiempo de enfermería y hasta el 52\% en estancias hospitalarias del presupuesto en salud de los países desarrollados ${ }^{2-4}$. A pesar de los avances en las mejores prácticas y la tecnología de los apósitos, la cifra de heridas complejas va en aumento, lo que plantea ciertos desafíos para el sistema de salud, incluido el uso indiscriminado de antibióticos con su incremento y resistencias asociadas 5 .

Las heridas complejas afectan a un gran porcentaje de la población, entre un $2 \%$ y un $6 \%{ }^{6}$. Como ejemplo podríamos mencionar que en el Reino Unido existen 2,2 millones de personas con heridas ${ }^{7}$ o que las camas hospitalarias de ese país se encuentran ocupadas entre un $25 \%$ y un $50 \%$ por pacientes con heridas 8 . Esto redunda en un gasto de millones de dólares ${ }^{9}$, que en Europa puede llegar a ser de entre el 2\% y el 4\% del gasto sanitario ${ }^{8,10}$.

Igualmente, la afectación de la calidad de vida de estos pacientes se ve asimilada a los que padecen enfermedad pulmonar obstructiva crónica y todas las repercusiones que tienen en estos pacientes en cuanto al dolor y su movilidad ${ }^{11}$. Así mismo, un reciente estudio ${ }^{12}$ afirma en sus conclusiones que existe una correlación entre el estado de la lesión y la calidad de vida; o el de Martín-Paradero et al. ${ }^{13}$ referido a la calidad de vida de los pacientes sometidos a diferentes procesos endovenosos de revascularización.

Si nos centramos únicamente en las UPP, solo en Espańa se pudieron gastar de media 602 millones de euros anuales ${ }^{2}$, incluyendo materiales y tiempo de enfermería, oscilando el coste por semana en atención primaria entre $27 €$ y $201 €$, dependiendo del estadio, a pesar de tener herramientas para la valoración del riesgo específicas para atención domiciliaria ${ }^{14,15}$ y poder prevenir este tipo de lesiones. Estas lesiones suponen más del $5 \%$ del gasto sanitario anual del país ${ }^{2}$. En el Servicio Nacional de Salud (NHS) inglés, entre los años 2012 y 2013 se estimó que el gasto en el cuidado de heridas y sus comorbilidades asociadas osciló entre 4,5 y 5,1 billones de libras anuales ${ }^{16}$. En otros países como Estados Unidos, el gasto para este tipo de lesiones puede rondar los 11000 millones de dólares al año ${ }^{17}$ y 25000 millones anuales para el tratamiento de heridas crónicas ${ }^{16}$.
Existe la tendencia a abordar los costes de las heridas primando el precio de los apósitos como la cuantía más importante del gasto total, cuando los estudios reflejan que solo suponen hasta un $22 \%{ }^{18}$. Por ello, las políticas han incidido en la reducción de los catálogos de productos disponibles ${ }^{19}$ y en la compra centralizada de los productos, creando plataformas de compras de materiales, mediante concursos provinciales en el caso de Andalucía o centralizados como en el caso del País Vasco ${ }^{19}$, constituidas en ocasiones por personas que no tienen contacto con heridas.

En el año 2017 se incorporó una aplicación informática unificada a nivel andaluz, dentro de DIRAYA (programa informático de historia digital de Andalucía), para el registro de heridas, y en el año 2018 se realizó un estudio del uso que realizaban de esta herramienta los profesionales en el Área de Gestión Sanitaria (AGS) Este de Málaga-Axarquía $^{20}$, donde se reflejaba la variabilidad en el abordaje de las heridas, como también reflejan otros estudios como el de Esperón Güimil et al. ${ }^{21}$. Desde ese mismo año 2017, El Grupo de Heridas Axarquía (GHA) optó por introducir, del inventario provincial, una serie de materiales en el catálogo del AGS para que los profesionales pudieran disponer de ellos en sus almacenes y consultas, evitando la prescripción de estos materiales, e intentar reducir la variabilidad en el uso de toda la gama disponible para su prescripción.

Por otro lado, la figura de enfermera de práctica avanzada (EPA) en heridas crónicas complejas (HCC) que se está desarrollando como experiencia en Andalucía ${ }^{22}$ no es del todo similar a la figura norteamericana $^{23}$ de enfermera especialista en heridas, ostomías e incontinencia y cuyo campo se amplió posteriormente a las heridas crónicas, o la figura del Reino Unido de enfermera especialista en viabilidad de tejidos ${ }^{24}$; en Andalucía se ha definido el perfil de competencias mediante un procedimiento específico a través de la Agencia de Calidad Sanitaria de Andalucía ${ }^{25}$. La Unidad de Gestión Clínica (UGC) donde se realiza este estudio dispone de un EPA en heridas crónicas complejas acreditado desde 2019.

Este artículo trata de mostrar que con la introducción de una nueva figura como la EPA en HCC y un nuevo modelo organizativo en las UGC de atención primaria se promueve un uso eficiente de los recursos, una mayor satisfacción y una mejor calidad de vida de los pacientes atendidos.

\section{Y OBJETIVOS}

- Analizar el coste económico que ha tenido el cierre de las heridas de la UGC de cada enfermero o enfermera comparado con la EPA de la Unidad.

- Contrastar la cantidad de heridas tratadas por cada enfermero o enfermera comparado con la EPA.

- Evaluar las heridas complicadas tratadas por cada profesional y la EPA.

- Comparar la media de días para el cierre de las heridas de cada enfermero o enfermera con la EPA. 


\section{\METODOLOGÍA}

\section{Tipo de estudio}

Estudio descriptivo longitudinal del total de los informes de valoración de heridas generados desde la aplicación de heridas en DIRAYA (sistema de información asistencial corporativo para todo el Servicio Andaluz de Salud, historia de salud única de todos los episodios asistenciales del ciudadano) y que necesitaron materiales de cura en ambiente húmedo suministrados por la UGC.

\section{Ámbito de estudio}

El estudio se desarrolló en la UGC de Algarrobo, perteneciente a la AGS Este de Málaga-Axarquía, desde junio 2019 hasta junio 2020.

La UGC está situada en una zona rural dispersa que consta de 5 consultorios locales, 2 auxiliares y un centro de salud, que son atendidos por 11 profesionales de enfermería, incluida la EPA, con una población de 17129 habitantes distribuidos en 8 núcleos principales y numerosos diseminados. El 22\% son habitantes mayores de 65 años.

\section{Unidad de estudio}

- Todas las hojas registradas en la aplicación de heridas de DIRAYA y que necesitaron materiales de cura en ambiente húmedo suministrados por la UGC.

- Los 11 profesionales de enfermería que atienden a sus cupos correspondientes, incluida la EPA en HCC.

- Se trata de una muestra conceptual, ya que se ha incluido a toda la población de enfermeros y enfermeras de la UGC y las correspondientes hojas emitidas por ellos.

Criterios de inclusión: las hojas de valoración de heridas de cualquier etiología que necesitan materiales de cura en ambiente húmedo suministrado por la UGC.

Criterios de exclusión: no existen criterios de exclusión. Todas las valoraciones fueron analizadas.

\section{Variables analizadas}

Profesional responsable de la herida (numérico). Se le asigna un número a cada profesional.

Tipo de lesión. Viene definida en la aplicación de heridas: venosas, arteriales, UPP, etc.

Fecha de la lesión. Fecha en la que se atiende por primera vez a la persona con la herida.

Localización. Viene definida en la aplicación de heridas: pierna derecha, sacrocoxígea, abdomen, etc.

Producto 1 de cura en ambiente húmedo usado (si solo se ha usado un producto).

Producto 2 de cura en ambiente húmedo usado (si se han usado dos productos en la misma herida y a la vez).

Producto 3 de cura en ambiente húmedo usado (si se han usado tres productos en la misma herida y a la vez).

Cantidades del producto 1 . Número de unidades suministradas para la metodología de curas establecida.

Cantidades del producto 2. Número de unidades suministradas como segundo producto a aplicar en la herida además del primero.

Cantidades del producto 3. Número de unidades suministradas como tercer producto, además del primero y el segundo.
Valor del Resvech 2.0 en cada valoración de la herida para el suministro de material.

Estadio/grado de la lesión reflejado en la aplicación heridas, de 1 a 4.

Coste total de los productos suministrados en la fecha de cada valoración, según programa "siglo" del almacén del área sanitaria.

Fecha del alta de la herida.

Fecha de cierre.

Días transcurridos desde inicio de la herida y del suministro de material, hasta el alta en la aplicación de heridas.

\section{MÉTODOS E INSTRUMENTOS PARA LA RECOGIDA DE DATOS}

Una vez realizada la valoración de la herida y registrada en la aplicación de heridas del sistema DIRAYA, se imprime la hoja de valoración, donde se incluyen los materiales de cura en ambiente húmedo necesarios.

La hoja es remitida a través del circuito establecido en la UGC para los documentos de carácter confidencial por el profesional de enlace que realiza todos los días el circuito entre los consultorios, y es entregada a la EPA.

La hoja es analizada por la EPA y registrada en una base de datos. Se suministra el material solicitado o en su defecto el acordado entre ambos profesionales si la valoración de la EPA así lo requiriera.

El suministro de material se realiza en cantidad suficiente para 2 semanas, transcurridas las cuales se realiza una nueva valoración, con la emisión de una nueva hoja, y se continúa el procedimiento hasta el cierre de la herida.

\section{Análisis de los datos}

Se ha realizado un análisis descriptivo de todas las variables consideradas, expresándose las cualitativas como frecuencias y porcentaje y las cualitativas, como medias y su desviación estándar.

\section{Aspectos éticos}

Se garantizó la confidencialidad de los datos, no se utilizaron sus nombres, ni en el informe final, ni durante la codificación, análisis y difusión de los datos según la Ley Orgánica 3/2018, de 5 de diciembre, de Protección de datos personales y garantía de los derechos digitales (ley orgánica que tiene por objeto garantizar y proteger, en lo que concierne al tratamiento de los datos personales, las libertades públicas y los derechos fundamentales de las personas físicas, y especialmente de su honor e intimidad personal $y$ familiar).

\section{$\searrow$ RESULTADOS}

Desde la acreditación del profesional de enfermería como EPA en HCC y la puesta en funcionamiento del nuevo procedimiento de suministro de material mediante las hojas de valoración de la aplicación de heridas de DIRAYA, el registro de heridas respecto al periodo anterior al del estudio aumentó en un $85 \%$. Durante este periodo se registraron un total de 416 heridas en la Unidad, de las cuales se cerraron 338, el 81,2\%.

En la tabla 1 se muestra el número de heridas tratadas por cada profesional, con 94 heridas tratadas por la EPA, con una desviación estándar de 22,1. También se muestra el número de heridas cerradas, que en el caso de la EPA es de 85 , con una resolución del $90,4 \%$, siendo la media de 30,7 con una desviación de 21,4. 
Tabla 1. Número total de heridas por profesional, porcentaje respecto al total de las heridas de la Unidad, además del número de heridas cerradas y el porcentaje de consecución de cierre de las heridas, media y desviación estándar

\begin{tabular}{|l|c|c|c|c|}
\hline Profesional & $\begin{array}{c}\text { Total de } \\
\text { heridas }\end{array}$ & $\begin{array}{c}\text { Porcentaje } \\
\text { respecto a la UGC }\end{array}$ & $\begin{array}{c}\text { Heridas } \\
\text { cerradas }\end{array}$ & $\begin{array}{c}\text { Resolución de } \\
\text { las heridas (\%) }\end{array}$ \\
\hline Uno & 49 & 11,7 & 42 & 85,7 \\
\hline Dos & 19 & 4,5 & 13 & 68,4 \\
\hline Tres & 14 & 3,4 & 10 & 71,4 \\
\hline Cuatro & 30 & 7,2 & 23 & 76,6 \\
\hline Cinco & 30 & 7,2 & 27 & 90 \\
\hline Seis & 32 & 7,7 & 19 & 59,3 \\
\hline Siete & 30 & 7,2 & 23 & 76,6 \\
\hline Ocho & 57 & 13,7 & 50 & 87,7 \\
\hline Nueve & 32 & 7,7 & 22 & 68,7 \\
\hline Diez & 29 & 6,9 & 24 & 82,7 \\
\hline EPA & 94 & 22,6 & 85 & 90,4 \\
\hline Media (DE) & $37,8(22,1)$ & & $30,7(21,4)$ & \\
\hline
\end{tabular}

DE: desviación estándar; EPA: enfermera de práctica avanzada; UGC: unidad de gestión clínica.
Se analizó el tipo de heridas más complicadas cerradas, tratadas por cada profesional. En la tabla 2 se detallan las heridas. La EPA trató 44 heridas, con una desviación estándar de 11, entre lesiones relacionadas con la dependencia, tumorales, relacionadas con problemas circulatorios y las propias del pie de los diabéticos.

Los costes en materiales de cura en ambiente húmedo utilizados por la EPA ascendieron a $1813,59 €$ en el ańo de estudio, por el alto volumen de heridas tratadas. La media de todos los profesionales fue de $935,15 €$ con una desviación de 549,5. Por otro lado, sin embargo, el coste medio de la EPA fue de $21,3 €$ para el cierre de la herida, siendo el coste medio general de 34,91€ con una desviación de 17,7 (tabla 3).

Lo más destacable es la media de días para el cierre de las heridas, que en el caso de la EPA fue de 30,7 días, mientras que la media general arroja una cifra de 159,27 días, con una desviación de 111,7 (tabla 3).

En la tabla 4 se ha querido mostrar las heridas venosas, por su dificultad para cerrarlas si no se usan los materiales adecuados como la compresión. La EPA trató 4 heridas por su trayectoria de larga evolución, media general de 2,36 con una desviación de 1,86, donde destaca que el coste medio para el cierre fue de $6,5 €$, mientras que el general fue de $60,1 €$ con una desviación de 76. Igualmente, es de reseñar que la EPA cerro las úlceras venosas en 98 días de media, respecto a los 208,5 días de media general, con una desviación de 242,3.

Tabla 2. Heridas más complicadas cerradas, tratadas por cada profesional, su media y desviación estándar

\begin{tabular}{|l|c|c|c|c|c|c|c|c|c|c|c|c|c|}
\multicolumn{1}{|c|}{ Profesional } & $\mathbf{1}$ & $\mathbf{2}$ & $\mathbf{3}$ & $\mathbf{4}$ & $\mathbf{5}$ & $\mathbf{6}$ & $\mathbf{7}$ & $\mathbf{8}$ & $\mathbf{9}$ & $\mathbf{1 0}$ & EPA & Media & DE \\
\hline Tumorales & $\mathbf{0}$ & $\mathbf{0}$ & $\mathbf{0}$ & $\mathbf{0}$ & $\mathbf{0}$ & $\mathbf{0}$ & $\mathbf{0}$ & 2 & 0 & 0 & 3 & 0,45 & 1,04 \\
\hline Venosas & 2 & 0 & 1 & 1 & 6 & 2 & 4 & 3 & 3 & 0 & 4 & 2,36 & 1,86 \\
\hline LRCD & 11 & 1 & 4 & 9 & 6 & 7 & 2 & 5 & 10 & 7 & 19 & 7,36 & 4,97 \\
\hline Arteriales & 0 & 0 & 0 & 0 & 0 & 0 & 0 & 0 & 1 & 0 & 8 & 0,82 & 2,4 \\
\hline Neuroisquémicas & 0 & 3 & 0 & 0 & 0 & 0 & 0 & 0 & 0 & 0 & 4 & 0,64 & 1,43 \\
\hline Sinus & 1 & 0 & 0 & 1 & 0 & 0 & 2 & 0 & 0 & 0 & 2 & 0,55 & 0,82 \\
\hline Neuropáticas & 0 & 0 & 0 & 0 & 0 & 1 & 1 & 0 & 0 & 0 & 4 & 0,55 & 1,21 \\
\hline Total de heridas complicadas & 14 & 4 & 5 & 11 & 12 & 10 & 7 & 10 & 14 & 7 & 44 & 12,5 & 11 \\
\hline
\end{tabular}

DE: desviación estándar; LRCD: lesiones relacionadas con la dependencia.

Tabla 3. Coste total de apósitos para todas las heridas cerradas, coste medio del cierre de cada herida y media de días para el cierre de una herida, media y desviación estándar

\begin{tabular}{|c|c|c|c|c|}
\hline Profesional & Coste total en apósitos & $\begin{array}{l}\text { Coste medio del cierre } \\
\text { de cada herida }\end{array}$ & $\begin{array}{c}\text { Media de días para el cierre } \\
\text { de la herida }\end{array}$ & Heridas cerradas \\
\hline Uno & $518 €$ & $12,3 €$ & 60,7 & 42 \\
\hline Dos & $317,72 €$ & $22,7 €$ & 257,6 & 13 \\
\hline Tres & $450,23 €$ & $45,02 €$ & 160,8 & 10 \\
\hline Cuatro & $523,58 €$ & $22,7 €$ & 139,4 & 23 \\
\hline Cinco & $776,74 €$ & $28,7 €$ & 115 & 27 \\
\hline Seis & $810,01 €$ & $45 €$ & 268,6 & 19 \\
\hline Siete & $959,49 €$ & $41,7 €$ & 376,9 & 23 \\
\hline Ocho & $1698,9 €$ & $34,7 €$ & 65,6 & 50 \\
\hline Nueve & $1715,79 €$ & $78 €$ & 231,9 & 22 \\
\hline Diez & $702,64 €$ & $31,9 €$ & 44,8 & 24 \\
\hline EPA & $1813,59 €$ & $21,3 €$ & 30,7 días & 85 \\
\hline Media (DE) & $935,15(549,5)$ & $34,91(17,7)$ & $159,27(111,7)$ & $30,73(21,4)$ \\
\hline
\end{tabular}

DE: desviación estándar; EPA: enfermera de práctica avanzada. 
Tabla 4. Número de heridas venosas, coste medio para el cierre y número medio de días para el cierre, media y desviación estándar

\begin{tabular}{|l|c|c|c|}
\hline Profesional & Venosas & $\begin{array}{c}\text { Coste medio del } \\
\text { cierre de la herida (€) }\end{array}$ & $\begin{array}{c}\text { Media de días para } \\
\text { el cierre de la herida }\end{array}$ \\
\hline Uno & 2 & 29,5 & 129,5 \\
\hline Dos & 0 & 0 & 0 \\
\hline Tres & 1 & 245,49 & 592 \\
\hline Cuatro & 1 & 62,45 & 135 \\
\hline Cinco & 6 & 19,5 & 105,6 \\
\hline Seis & 2 & 123,5 & 334,5 \\
\hline Siete & 4 & 124,3 & 730 \\
\hline Ocho & 3 & 31,44 & 81,7 \\
\hline Nueve & 3 & 18,47 & 87,7 \\
\hline Diez & 0 & 0 & 0 \\
\hline EPA & 4 & 6,5 & 98 \\
\hline Media (DE) & $2,36(1,86)$ & $60,1(76)$ & $208,5(242,3)$ \\
\hline
\end{tabular}

DE: desviación estándar; EPA: enfermera de práctica avanzada.

De la misma manera, en la tabla 5 se ha querido mostrar las lesiones relacionadas con la dependencia cerradas, por su alta merma en la calidad de las personas que las padecen, tratando la EPA 19 lesiones por ser las más complicadas, siendo la media de 7,4 lesiones con una desviación de 5 y con un coste medio para el cierre por la EPA de 62,1€ y una media de 43,4 con una desviación de 35,9. Pero al igual que sucede con las úlceras venosas, los días para el cierre de las lesiones relacionadas con la dependencia por la EPA fue de 33,3 de media, mientras que la media era de 104,8 días, con una desviación de 90,3.

\section{У DISCUSIÓN}

El presente estudio fue diseñado para mostrar que con la introducción en las UGC de atención primaria de una nueva figura como la EPA en HCC y un nuevo modelo organizativo se promueve un uso eficiente de los recursos y una mejor calidad de vida de los pacientes atendidos. Así mismo, ofrece una visión de la situación en la UGC de Algarrobo, del análisis de los datos de las heridas en general y de las complejas en particular, tratadas por la EPA en HCC y el resto de profesionales de enfermería.

Desde que el enfermero de la UGC se acreditó como EPA en HCC, se introdujo un nuevo método de suministro de material de curas en ambiente húmedo, basado en la aplicación de heridas de DIRAYA. Se han realizado numerosas actividades docentes con los profesionales de la Unidad y se les ha suministrado información con la evidencia actualizada, además de datos correspondientes a sus heridas y evolución. Se implantó un circuito de consulta y visitas a usuarios para establecer objetivos conjuntos.

Encontramos una gran dificultad a la hora de comparar estos resultados con otros que sean similares, ya que la EPA en HCC es una figura reciente en España y sin datos con los que comparar al no encontrar estudios semejantes.

En el año analizado, se ha realizado el seguimiento de 416 personas con lesiones, de las cuales 144 se catalogan como complicadas; en el periodo anterior al estudio (mayo 2018-mayo 2019) se registraron 224 heridas, aumentando el registro de las lesiones durante el periodo de estudio en
Tabla 5. Número de lesiones relacionadas con la dependencia, coste medio para el cierre, número medio de días para el cierre, media y desviación estándar

\begin{tabular}{|l|c|c|c|}
\hline Profesional & LRCD & $\begin{array}{c}\text { Coste medio del } \\
\text { cierre de la herida (€) }\end{array}$ & $\begin{array}{c}\text { Media de días para el } \\
\text { cierre de la herida }\end{array}$ \\
\hline Uno & 11 & 10,6 & 67,5 \\
\hline Dos & 1 & 15,36 & 19 \\
\hline Tres & 4 & 23,8 & 54,25 \\
\hline Cuatro & 9 & 38,4 & 83,5 \\
\hline Cinco & 6 & 56,3 & 142,7 \\
\hline Seis & 7 & 51,6 & 235,6 \\
\hline Siete & 2 & 11,16 & 16 \\
\hline Ocho & 5 & 39,2 & 185 \\
\hline Nueve & 10 & 137,3 & 272,1 \\
\hline Diez & 7 & 31,38 & 43,7 \\
\hline EPA & 19 & 62,1 & 33,3 \\
\hline Media (DE) & $7,4(5)$ & $43,4(35,9)$ & $104,8(90,3)$ \\
\hline
\end{tabular}

DE: desviación estándar; EPA: enfermera de práctica avanzada; LRCD: lesiones relacionadas con la dependencia.

un $85 \%$. Así mismo, la resolución de las heridas se incrementó un $20 \%$ respecto al periodo anterior.

En esta Unidad se ha tratado y realizado seguimiento del 18,7\% de las lesiones complejas del total de las lesiones complejas tratadas en cuatro distritos andaluces, según los datos de un estudio publicado por Jiménez García et al. ${ }^{26}$. Estos pacientes y el sistema sanitario se han beneficiado de un mejor tratamiento clínico, una reducción de costes, -siendo el coste medio del cierre de una herida de $24,7 €$ en el periodo de estudio y de $51,5 €$ en el periodo anterior- y una mejor tasa de cicatrización ${ }^{27-29}$, sin dejar de lado los beneficios ante el dolor y la calidad de vida de estos pacientes ${ }^{12,30}$.

Destacamos que el coste medio para el cierre de una herida complicada por la EPA es de 21,3€, mientras que en unidades especiales donde se tratan heridas crónicas, el coste medio de cada cura, no hablamos del cierre, es de $7,05 \pm 3,43^{31}$.

En los cuatro distritos de Andalucía donde existe una EPA en HCC se ha conseguido reducir el consumo en un $64 \%$ en 2 ańos ${ }^{32}$. En nuestra unidad se ha conseguido bajar al $21 \%$ en un ańo, seguramente a causa de la mejor adecuación de los tratamientos guiados por la EPA, a la mejor formación de los profesionales y mejores medidas preventivas en los domicilios.

Si nos detenemos en las heridas de origen venoso, observamos la alta resolución de las mismas debido al uso de sistemas de compresión adecuados por la EPA y los profesionales que han sido adiestrados para ello. Igualmente, el coste del cierre de la herida venosa por la EPA y el tiempo de resolución es notablemente significativo, mejorando la calidad de vida de las personas atendidas. Lo mismo sucede con las lesiones relacionadas con la dependencia, donde se incluyen las UPP, siendo claramente diferente en favor de la EPA.

Como aspecto a mejorar está el tiempo transcurrido hasta el cierre de una herida, ya que existen grandes diferencias entre la EPA y los profesionales.

Está previsto realizar una nueva revisión transcurrido un año, durante el cual y previo al estudio se seguirá manteniendo la docencia en áreas específicas como el vendaje compresivo o las características técnicas de los materiales de cura en ambiente húmedo de los que se disponen en el catálogo del área sanitaria. También durante el mismo periodo se establecerá un turno para que cada profesional rote por la consulta de la EPA en HCC. 


\section{$У$ CONCLUSIONES}

- Valorando el coste económico de cada herida una vez cerrada, se constata que las heridas tratadas por la EPA han tenido un coste menor, a pesar de tener mayor número de heridas complicadas que el resto de profesionales de enfermería.

- La EPA ha tratado una cuarta parte del total de las heridas de la Unidad, acercándose al cierre total de todas las heridas tratadas.

- La mitad de las heridas complicadas de la Unidad que han sido resueltas han sido tratadas por la EPA.

- La media de días para el cierre de las heridas tratadas por la EPA está muy por debajo del resto de profesionales de enfermería.

La introducción de la EPA en HCC en la UGC ha llevado a la Unidad a un mejor resultado en cuanto a gasto en materiales y al acorta- miento de los días para la resolución de las heridas, lo que influye en una mejor calidad de vida para aquellos que las padecen.

Además, la introducción de la EPA ha servido de revulsivo para los profesionales y les ha llevado a un mejor hacer en su trabajo con las heridas, lo que influye tanto en su autoestima profesional como en los pacientes a los que tratan

\section{Agradecimientos}

A todos los profesionales de la UGC de Algarrobo, por su colaboración y su buen hacer diario.

\section{Conflicto de intereses}

Los autores declaran no tener conflicto de intereses.

\section{Y BIBLIOGRAFÍA}

1. European Wound Management Association (EWMA). Documento de Posicionamiento: Heridas de difícil cicatrización: un enfoque integral. Londres: MEP; 2008

2. Soldevilla Agreda JJ, Torra i Bou J-E, Posnett J, Verdú Soriano J, San Miguel L, Mayan Santos JM. Una aproximación al impacto del coste económico del tratamiento de las úlceras por presión en España. Gerokomos. 2007;18(4):201-10.

3. Drew P, Posnett J, Rusling L, on behalf of the Wound Care Audit Team. The cost of wound care for a local population in England. Int Wound J. 2007:4:149-55.

4. Vowden K, Vowden P, Posnett J. The resource costs of wound care in Bradford and Airedale primary care trust in the UK J Wound Care 2009:18:93-4, 96-8.

5. Murphy C, Atkin L, Swanson T, Tachi M, Tan YK, Vega de Ceniga $M$, et al. International consensus document. Defying hard-toheal wounds with an early antibiofilm intervention strategy: wound hygiene. J Wound Care. 2020;29(Suppl 3b):S1-8.

6. Järbrink K, Ni G, Sönnergren H, Sönnergren H, Schmidtchen A, Pang $C$, et al. The humanistic and economic burden of chronic wounds: a protocol for a systematic review. Syst Rev 2017:6:15.

7. Campbell D. Chronic wounds: the hidden health crisis hitting 2m Britons. The Guardian. 2019 July 29. Disponible en: https:// www.theguardian.com/lifeandstyle/2019/jul/29/uncomfortable-ugly-painful-the-hidden-health-crisis-hitting-2m-britons (acceso 22 de junio 2020).

8. Posnett J, Gottrup F, Lundgren H, Saal G. The resource impact of wounds on health-care providers in Europe. J Wound Care. 2009:18:154-61.

9. Nussbaum SR, Carter MJ, Fife CE, DaVanzo J, Haught R, Nusgart $M$, et al. An economic evaluation of the impact, cost, and medicare policy implications of chronic nonhealing wounds. Value in Health. 2018;21:27-32.

10. Purwins S, Herberger K, Debus ES, Rustenbach SJ, Pelzer P, Rabe $\mathrm{E}$, et al. Cost-of-illness of chronic leg ulcers in Germany. Int Wound J. 2010;7:97-102.

11. Olsson M, Järbrink K, Divakar U, Bajpai R, Upton Z, Scmidtchen $A$, et al. The humanistic and economic burden of chronic wounds: A systematic review. Wound Repair Regen. 2019;27:114-25.
12. Perdomo-Pérez E, Soldevilla-Agreda J, García-Fernández FP. Relación entre calidad de vida y proceso de cicatrización en heridas crónicas complicadas. Gerokomos. 2020:31(3):166-72.

13. Martín-Paredero V, Arrébola-López M, Berga-Fauria C, PañeIla-Agustí F, Gómez-Moya B. Impacto en la calidad de vida. Angiologia. 2003:55(1):250-9.

14. García-Díaz FJ, Cabello-Jaime R, Muñoz-Conde M, Bergera-Lezaun I, Blanca-Barba F, Carrasco-Herrero JM, et al. Validación de las escalas de Braden y EMINA en pacientes de atención domiciliaria incluidos en programa de inmovilizados. Gerokomos. 2015;26(4):150-6.

15. García-Díaz FJ, Cabello-Jaime R, Muñoz-Conde M, Bergera-Lezaun I, Blanca-Barba F Carrasco-Herrero JM, et al. Fiabilidad de las escalas de Braden y EMINA en pacientes de atención domiciliaria incluidos en programa de inmovilizados. Gerokomos. 2014;25(3):124-30

16. Vowden P. Vowden K. The economic impact of hard-to hea wounds: promoting practice change to address passivity in wound management. Wounds Int. 2016;7(2).

17. Sen CK, Gordillo GM, Roy S, Kirsner R, Lambert L, et al. Human skin wounds: a major and snowballing threat to public health and the economy. Wound Repair Regen. 2009;17(6):763-71.

18. Carrión Jiménez A, Núñez Ortiz C, Gallego Galisteo M, Rodríguez Cardoso R, Sánchez Meléndez A. Coste-eficiencia de la elección de apósitos en heridas crónicas con biofilm basado en un modelo teórico. Gerokomos. 2017;28(2):98-102.

19. Quesada Ramos C, Beistegui A. Evaluación económica de un nuevo modelo de compra centralizada de apósitos: el caso del País Vasco. Gerokomos. 2014;25(3):118-23

20. García-Díaz FJ, Cabello-Jaime R, Muñoz-Conde M. Evaluación del uso de una herramienta informática para valoración y seguimiento de heridas. $10^{\circ}$ Congreso virtual internacional de enfermería y fisioterapia "Ciudad de Granada". Innovación y mejoras en los cuidados. Avances y Tecnologías en salud. 2019.

21. Esperón J, Rodríguez M, Fuentes V, Fernández J, García I, SoldeviIla-Agreda J. Variabilidad en el abordaje de las heridas crónicas: ¿qué opinan las enfermeras? Gerokomos. 2014;25(4):171-10.

22. Jiménez García JF, Aguilera Manrique G, Arboledas Bellón J, Gutiérrez García M, González Jiménez F, García Fernández FP.
Efectividad de la enfermera de práctica avanzada en el cuidado de los pacientes con úlceras por presión en atención primaria. Gerokomos. 2019:30(1):28-33.

23. Beitz JM. Specialty practice, advanced practice, and WOC nursing: current Professional issues and future opportunities Wound Ostomy Continence Nurs. 2000;27(1):55-64.

24. Haughton B. The formation of a new tissue viability nurses group. Br J Nurs. 2000;9(19 Suppl):S4.

25. Agencia de Calidad Sanitaria de Andalucía. Manual de competencias profesionales. Enfermero/a de Práctica Avanzada en Cuidados a Personas con Heridas Crónicas Complejas. Sevilla: Junta de Andalucía, Consejería de Salud; 2017.

26. Jiménez García JF, Aguilera Manrique G, González Jiménez F, Gutiérrez García M, Arboledas Bellón J, García Fernández FP. Resultados clínicos de las enfermeras de práctica avanzada en heridas crónicas complejas en Andalucía. Gerokomos. 2020;31(1):36-40.

27. Guest JF, Fuller GW, Vowden P. Venous leg ulcer management in clinical practice in the UK: costs and outcomes. Int Wound J. 2018;15(1):29-37.

28. Guest JF, Fuller GW, Vowden P. Diabetic foot ulcer management in clinical practice in the UK: costs and outcomes. Int Wound J. 2018;15(1):43-52

29. Demarré L, Van Lancker A, Van Hecke A, Verhaeghe S, Grypdonck $M$, Lemey J, et al. The cost of prevention and treatment of pressure ulcers: A systematic review. Int J Nurs Stud. 2015:52(11):1754-74.

30. Newbern S. Identifying Pain and Effects on Quality of Life from Chronic Wounds Secondary to Lower-Extremity Vascular Disease: An Integrative Review. Adv Ski Wound Care. 2018;31(3):102-8.

31. Ramos A, Morillo JM, Gayo N, Tasiguano JE, Munzón E, Ribeiro ASF. Curar o paliar: ¿qué cuesta más? Análisis de costes de tratamiento de una herida crónica en función de su finalidad. Medicina Paliativa. 22(2):45-51.

32. Jiménez-García JF, Arboledas-Bellón JF, Ruiz-Fernández C Gutiérrez-García M, Lafuente-Robles N, García-Fernández FP. La enfermera de práctica avanzada en la adecuación de los

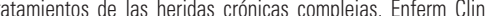
2019;29(2):74-82. 